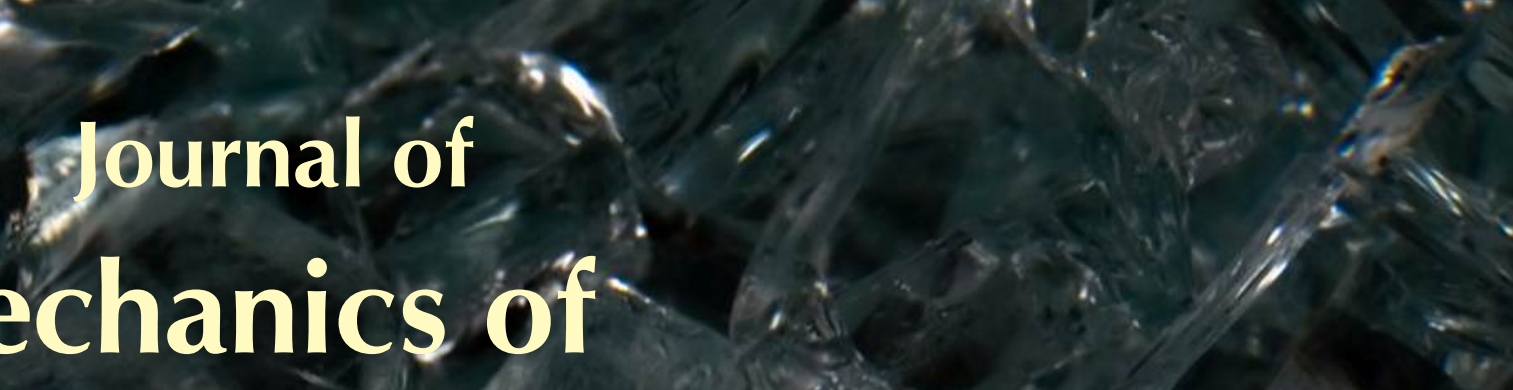

Materials and Structures

Mechanics of
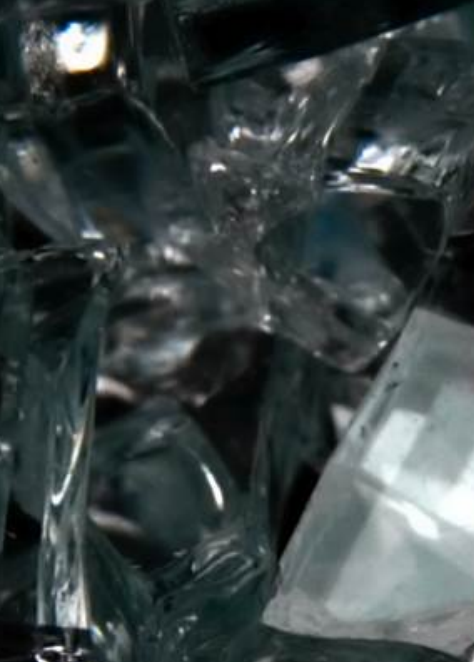

बiर

1. CHARACTERIZATION OF HUMAN SKIN THROUGH SKIN EXPANSION

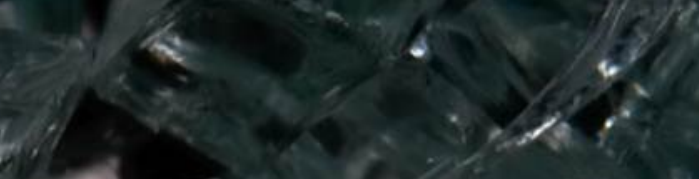

1

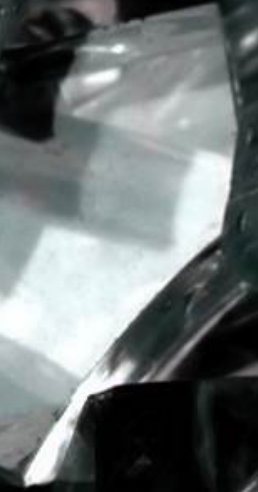

Djenane C. Pamplona and Claudio R. Carvalho

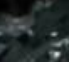

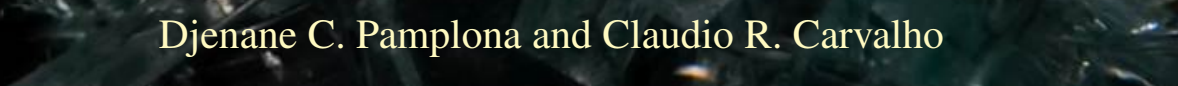




\title{
CHARACTERIZATION OF HUMAN SKIN THROUGH SKIN EXPANSION
}

\author{
Djenane C. Pamplona and Claudio R. Carvalho
}

\begin{abstract}
This study characterized human skin of the lower leg and scalp during the surgical process of skin expansion. To our knowledge, this is the first study in this field, which has provided results that considerably improve our understanding of human skin. A detailed in vivo analysis was carried out involving four different patients that allowed for observation during the relaxation process. A comparison between the in vivo and numerical finite elements model of the expansion was used to identify the material elastic parameters of the skin. After a comprehensive search of constitutive equations for describing skin, Delfino's constitutive equation was chosen to model the in vivo results. We considered skin as an isotropic, homogeneous, hyperelastic, and incompressible membrane. The parameters of Delfino's exponential function obtained for the first skin stretch process were $a=40.0 \mathrm{KPa}$ and $b=20.2$. As skin is extended, such as with expanders or in other procedures that tighten the skin, the collagen fibers are also extended and cause stiffening in the skin, which results in it being more and more resistant to expansion or further stretching. We observed this phenomenon as an increase in parameters $a$ and $b$ as subsequent expansions continued. The results of this study allow for the quantification of stiffening of the skin after several stretches, when the skin becomes more and more inelastic. These results are very encouraging and provide insight into our understanding of the behavior of stretched skin and maybe other biological tissues, as swollen artery and veins.
\end{abstract}

\section{A list of symbols can be found on page 653.}

\section{Introduction}

Skin expansion is a physiological process that is defined as the ability of human skin to increase its surface area in response to stress or deformation. Skin expanders are silicone bags of different shapes and sizes that are implanted under the skin. Since the skin presents creep or relaxation, the resulting stress decreases after an imposed deformation over a specific period of time. The physiology of skin expansion not only considers stretching of the skin, but also the relaxation process used to obtain an extra flap of skin that possesses the needed characteristics. For example, skin expansion is used to reconstruct burned areas and breasts after mastectomy and to hide scars. Expansion is usually conducted near the location where the skin is required in order to provide skin of the same color, texture, sensation, and structure as the one to be removed.

Starting in 1982, several studies have improved the expansion process using self-inflation, continuous tissue, or controlled expansion [Austad and Rose 1982; Schmidt et al. 1991; Duffy and Shuter 1994]. Other studies have explored concepts and complications of the surgical process, and numerous analyses have been conducted on skin expansion from a medical point-of-view. In contrast, few studies have

Keywords: characterization of human skin, finite elements, skin expansion, biomembranes, constitutive equation. 
explored the bioengineering process of expansion. In order to model reconstructive plastic surgeries numerically and achieve a better understanding of the process, it is critical to determine the mechanical properties of skin in vivo, and several recent studies have been performed with this goal in mind. The most frequently used techniques are indentation and suction of the skin, and some studies have used Young's modulus to establish the stiffness of the skin [Diridollou et al. 2000; Zahouani et al. 2009; Pailler-Mattei et al. 2009]. In addition, more recent studies have characterized the nonlinear mechanical behavior of human skin under indentation and suction processes [Hendriks et al. 2003; Delalleau et al. 2006; 2008; Geerligs et al. 2011; Lim et al. 2008].

To date, only one study has used a numerical model for skin under expansion [Socci et al. 2007], which assessed the stresses and strains of skin from inflation of an expander. This study only considered an axially symmetric configuration, in which a flat circular flap of a thin membrane (the skin) is expanded by a spherical balloon. In that study, a phenomenological approach was used to represent the growth of the skin after expansion.

The analysis of membranes under large deformation is usually based on the pioneering work of Green and Adkins in nonlinear elasticity [Green and Adkins 1970]. Several theoretical and numerical studies have been published in this field, but the total number of studies that have used this approach is rather low compared to theoretical and numerical approaches [Alexander 1971; Pamplona et al. 2006; 2001; Pamplona and Bevilacqua 1992; Gonçalves et al. 2009; 2008].

In this study, the skin was considered a hyperelastic membrane, and in order to execute the numerical analysis, finite membrane elements were used, since membrane structures are load adaptive and change their geometry to accommodate external loads with minimum variation in stress levels, and as the skin wrinkles in-plane compression. Our aim was to develop a method for characterizing the nonlinear mechanical behavior of skin under expansion using a numerical and in vivo technique. A consistent constitutive equation for the skin may allow for preoperative planning and optimal filling parameters in terms of both the volume of fluid expansion and duration between fills. Currently pain and observed tightness are the main guides to filling.

The study presented here aimed to model the human skin over successive skin expansions in order to obtain different parameters for the characterization of stretched skin over time. To accomplish this goal, a detailed in vivo analysis was carried out and at least four expansions were monitored for each patient, obtaining similar data for the same methodology done in animals [Beauchene et al. 1989]. During each expansion, at least five measurements were taken related to the volume inserted and the pressure inside the skin expander. These data provided enough information to characterize the skin at each stage of the stretching process, and the entire process provided more than 100 in vivo calculations of volume $\times$ pressure. To obtain a constitutive equation that could describe the measured skin, several well-known constitutive relationships were analyzed using several previously described methods [Ogden 2003; Holzapfel et al. 2000; Delfino et al. 1997]. Since the pressure inside the skin expander dropped to zero between expansions, the skin was also considered viscoelastic. Skin growth was not considered although an overview on the mechanobiology underlying skin growth sustains that tensile stress applied to skin appears to stimulate skin growth [Silver et al. 2003]. Researchers recently are looking for a model to describe the growth of the skin under tensile stress, establishing computational models for stretch-induced skin growth under expansion [Buganza Tepole et al. 2011] and also patient-specific finite element simulation of skin growth in situ [Zöllner et al. 2012]. 


\section{Methodology}

The first step for expanding skin during surgery was to implant a skin expander directly underneath the skin and subcutaneous tissue. The surgeon outlined the shape and size of the skin expander on the skin and then made an incision on one side of the outline. The pocket that will contain the expander is then made by separating the subcutaneous tissue by obeying the contour of the drawing, and therefore delimiting the flap of skin to be expanded. Through this incision, the surgeon inserted the skin expander under the skin together with a valve that is connected to the expander. After the incision is closed, a saline solution, which should be approximately $10 \%$ of the nominal volume of the skin expander, $V_{\text {exp }}$, was injected into the skin expander by inserting a needle into the implanted valve. At this stage the pressure inside the expander was not measured, and this step was designated as $A$. Fifteen days after the surgery, the expansion was initiated, which guarantees cicatrization. Each week, a specified volume of saline solution was inserted inside the expander, which was dependent on the size of the expander. As the solution was inserted, the skin expands due to the increased pressure inside the expander and results in some pain for the patient; however, due to viscoelastic properties, the skin relaxes over time, which diminishes the pressure inside the expander and consequently the pain of the patient. After one week, no measurable pressure exists inside the expander. The major disadvantage of this process is the need for two surgeries, where one is used to implant the expander and the other is used to remove the expander and repair the skin defect. This research was done on patients that needed, for medical reasons, skin expansion, thus the selection of patients for this analysis did not consider the age of the patient or region of the body to be expanded, though these can prove to be important factors, specially the relation with the original skin laxity of the anatomic region.

In addition, expansion over elastic foundations, such as the abdomen or fatty tissue, was not considered since these locations would compromise the results of the numerical model.

\section{In vivo analysis}

This study was conducted on four patients, and all patients signed an informed consent. Of those, two patients had skin expansions performed in the scalp and the other two in the lower leg. In order to identify the behavior of the skin from successive skin expansions, it was necessary to measure the pressure inside the skin expander before, during, and after injection of saline solution for each expansion. For this purpose, an apparatus was developed that provided a pressure sensor coupled to the syringe used to perform the injection of liquid (see figure). The apparatus was coupled to a plastic Y tube, where one upper region of the $\mathrm{Y}$ tube (b) was attached to the syringe with the saline solution to be injected, and the other upper region of the tube (c) was coupled to the apparatus developed to measure the pressure. In addition, the lower side of the Y tube (a) was attached to the needle used to inject the saline solution into the valve of the skin expander. The initial thickness of the skin, $H$, of each patient and region was determined by the surgeon after performing the surgery.

During each skin expansion, the surgeon paused after each 5 or $10 \mathrm{ml}$ of liquid was injected to measure the pressure inside of the expander, which provided both volume and pressure data points.

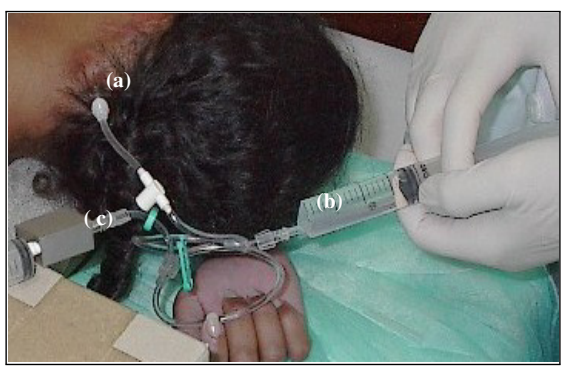

(a) needle; (b) syringe; (c) apparatus. 
The measurements were continued in this manner until the final volume of the expansion was reached. The curves relating volume to the pressure of each skin expansion allowed for characterization of the skin for that expansion. During the week following each expansion, the viscoelastic properties of the skin allowed for relaxation to occur and for the pressure inside the expander to reach zero.

Only the final pressure and volume reached in each expansion are shown in the following tables, although to achieve the final pressure each expansion was carefully measured. For these analyses, $V_{i-1}$ represented the initial volume and $V_{i}$ represented the final volume of the $i$-th expansion. The letter $A$ designated the initial insertion of fluid during the surgery, and the letter $B$ indicated that the pressure for that particular expansion could not be measured for various reasons (e.g., the patient arriving late or at times when the measurements could not be performed).

Since the skin expanders for the patients have different shapes, dimensions, and nominal volumes, $V_{\exp }$, the variable $V_{i}^{*}$ was used to relate to the final pressure of that expansion, $P_{i}$. The ratio between $V_{\exp }$ and the final volume $V_{i}$ inside the expander at the end of each expansion, $i$, is denoted by

$$
V_{i}^{*}=\frac{V_{i}}{V_{\exp }}
$$

It is important to note that although the volume injected into the expander could be the same in each step, this volume is a smaller percentage of the total volume already injected into the expander, which is

$$
V_{i}^{* *}=\frac{V_{i}-V_{i-1}}{V_{i-1}}
$$

3.1. Results of skin expansion in the scalp. Two patients were analyzed for expansion of the skin in the scalp, both with initial skin thickness $H=0.5 \mathrm{~cm}$. Patient 1 was a 33-year-old female who had light brown skin, weighed $53 \mathrm{~kg}$, and was $1.60 \mathrm{~m}$ in height. A rectangular skin expander $\left(V_{\exp }=400 \mathrm{ml}\right)$ was used on this patient. Patient 2 was a 12-year-old female with white skin, weighed $35 \mathrm{~kg}$, and was $1.50 \mathrm{~m}$ in height. A lunar crescent-shaped expander $\left(V_{\exp }=300 \mathrm{ml}\right)$ was used on this patient.

Since the pressure inside the expander was measured after every 5 or $10 \mathrm{ml}$ of liquid was injected, at least 4 parameters of the inside pressure related to the injected volume were determined for each skin expansion $(i)$ obtained. Tables 1 and 2 show only the final results of the maximum pressure reached at the end of each weekly expansion $(i)$.

\begin{tabular}{|ccccccc|}
\hline (i) & $V_{i-1}(\mathrm{ml})$ & $V_{i}(\mathrm{ml})$ & $V_{i}-V_{i-1}(\mathrm{ml})$ & $V_{i}^{*}$ & $V_{i}^{* *}$ & $P_{i}(\mathrm{kPa})$ \\
$\mathrm{A}$ & 0 & 80 & $\mathrm{x}$ & $\mathrm{x}$ & $\mathrm{x}$ & $\mathrm{x}$ \\
1 & 0 & 110 & 30 & 0.28 & 0.38 & 29.50 \\
2 & 110 & 140 & 30 & 0.35 & 0.27 & 28.20 \\
$\mathrm{~B}$ & 140 & 380 & $\mathrm{x}$ & $\mathrm{x}$ & $\mathrm{x}$ & $\mathrm{x}$ \\
3 & 380 & 425 & 45 & 1.06 & 0.12 & 26.10 \\
4 & 425 & 465 & 40 & 1.16 & 0.09 & 26.80 \\
5 & 465 & 500 & 35 & 1.25 & 0.08 & 26.00 \\
6 & 500 & 538 & 38 & 1.35 & 0.08 & 25.20 \\
\hline
\end{tabular}

Table 1. Patient 1 with a rectangular expander $\left(V_{\exp }=400 \mathrm{ml}\right)$ inserted under the scalp. 


\begin{tabular}{|ccccccc|}
\hline$(i)$ & $V_{i-1}(\mathrm{ml})$ & $V_{i}(\mathrm{ml})$ & $V_{i}-V_{i-1}(\mathrm{ml})$ & $V_{i}^{*}$ & $V_{i}^{* *}$ & $P_{i}(\mathrm{kPa})$ \\
$\mathrm{A}$ & 0 & 214 & $\mathrm{x}$ & $\mathrm{x}$ & $\mathrm{x}$ & $\mathrm{x}$ \\
1 & 214 & 254 & 40 & 0.85 & 0.19 & 26.00 \\
2 & 254 & 298 & 44 & 0.99 & 0.17 & 25.10 \\
3 & 298 & 338 & 40 & 1.13 & 0.13 & 23.90 \\
4 & 338 & 376 & 38 & 1.25 & 0.11 & 21.90 \\
\hline
\end{tabular}

Table 2. Patient 2 with a crescent expander $\left(V_{\exp }=300 \mathrm{ml}\right)$ inserted under the scalp.

The data indicated that a maximum pressure achieved during each skin expansion was of the same magnitude and ranged between $22-29 \mathrm{kPa}$. This maximum pressure was limited by the discomfort or pain felt by the patient, important information to the doctor performing the procedure.

3.2. Results of skin expansion in the lower leg. Two patients were analyzed for expansion of skin on the lower leg, both with initial skin thickness $H=0.8 \mathrm{~cm}$. Patient 3 was a 10 -year-old female with light brown skin, weighed $45 \mathrm{~kg}$, and had a height of $1.50 \mathrm{~m}$. A rectangular expander $\left(V_{\exp }=250 \mathrm{ml}\right)$ was used on this patient. Patient 4 was an 18-year-old female with white skin, weighed $70 \mathrm{~kg}$, and had a height of $1.80 \mathrm{~m}$. A rectangular expander $\left(V_{\exp }=400 \mathrm{ml}\right)$ was also used on this patient. Since the pressure inside the expander was measured after every 5 or $10 \mathrm{ml}$ of liquid was injected, at least 4 parameters of the inside pressure related to the injected volume were determined for each skin expansion $(i)$. Tables 3 and 4 show the final results of the maximum pressure obtained at the end of each weekly expansion $(i)$.

As seen in Tables 3 and 4, the results showed that the maximum pressure achieved during each skin expansion of the lower leg ranged from $9-19 \mathrm{kPa}$, which was lower than that obtained on the scalp. The

\begin{tabular}{|ccccccc|}
\hline$(i)$ & $V_{i-1}(\mathrm{ml})$ & $V_{i}(\mathrm{ml})$ & $V_{i}-V_{i-1}(\mathrm{ml})$ & $V_{i}^{*}$ & $V_{i}^{* *}$ & $P_{i}(\mathrm{kPa})$ \\
$\mathrm{A}$ & 0 & 157 & $\mathrm{x}$ & $\mathrm{x}$ & $\mathrm{x}$ & $\mathrm{x}$ \\
1 & 157 & 187 & 30 & 0.75 & 0.19 & 12.63 \\
2 & 187 & 232 & 45 & 0.99 & 0.24 & 13.00 \\
3 & 232 & 272 & 40 & 1.09 & 0.17 & 10.50 \\
4 & 272 & 302 & 30 & 1.21 & 0.11 & 9.00 \\
\hline
\end{tabular}

Table 3. Patient 3 with a rectangular expander $\left(V_{\exp }=250 \mathrm{ml}\right)$ inserted in the lower leg.

\begin{tabular}{|ccccccc|}
\hline (i) & $V_{i-1}(\mathrm{ml})$ & $V_{i}(\mathrm{ml})$ & $V_{i}-V_{i-1}(\mathrm{ml})$ & $V_{i}^{*}$ & $V_{i}^{* *}$ & $P_{i}(\mathrm{kPa})$ \\
$\mathrm{A}$ & 0 & 120 & $\mathrm{x}$ & $\mathrm{x}$ & $\mathrm{x}$ & $\mathrm{x}$ \\
1 & 120 & 160 & 40 & 0.40 & 0.33 & 17.20 \\
2 & 160 & 200 & 40 & 0.50 & 0.25 & 18.90 \\
3 & 200 & 240 & 40 & 0.60 & 0.20 & $\mathrm{x}$ \\
4 & 240 & 280 & 40 & 0.70 & 0.17 & 12.90 \\
5 & 280 & 320 & 40 & 0.80 & 0.14 & 12.60 \\
\hline
\end{tabular}

Table 4. Patient 4 with a rectangular expander $\left(V_{\exp }=400 \mathrm{ml}\right)$ inserted in the lower leg. 
maximum pressure was also limited by the discomfort or pain that the patient felt, since discomfort was accentuated in the skin of the lower leg.

In addition, we also observed that the amount of pressure needed to fill the expander diminished as it was filled for both the scalp and skin of the lower leg, despite the injected volume being the same at each step. This could be related to Laplace's law often cited by surgeons, mostly related to aneurismal growth. In addition, this volume was a smaller percentage of the volume already present inside the expander, $V_{i}^{* *}$.

\section{Numerical analysis}

To characterize the skin during expansion, it is necessary to model the procedure numerically, which can be accomplished using the commercial finite element software ABAQUS (version 6.8) together with the commercial program MATLAB. The goal was to identify an elastic constitutive equation that can characterize the skin of the scalp and lower leg during each expansion. However, the parameters of the constitutive equation for each region and expansion are not the same, since the collagen fibers of the stretched skin offer more resistance to expansion over the course of the expansion, as observed in [Lim et al. 2008].

To perform the numerical analysis, the finite element mesh used linear hybrid membrane quadrilateral or triangular finite elements (M3D4 or M3D3), depending on the geometry, when the initial thickness, $H$, was provided by the surgeon. The control of the volume inserted into the skin expander is essential for modeling this medical procedure, and it can only be done in ABAQUS by using the combination of membrane and fluid finite elements. In the fluid elements, the pressure is applied to one unique node, which is called the reference of cavity node. This pressure simulates the injection of fluid into the skin expander. The middle surface is the reference point for both the membrane and fluid elements. In the numerical analysis, the final geometry of one expansion is used as the initial geometry for the next expansion where the stress and pressure are equal to zero, since the pressure inside goes to zero within a week due to the relaxation of the skin. Because the expansions are successive, the thickness of the modeled skin changes at the end of each expansion, but not uniformly. Modeling a different thickness for every membrane element is impossible, since finite elements of the membrane were used and require a constant thickness continuum. Therefore, the mean thickness of all elements obtained in the previous expansion was used for all subsequent expansions together with the previous geometry. The ABAQUS code has a command to obtain the thickness of each finite element after it is deformed at the end of each expansion. With a maximum stretch of the skin, it is possible to determine the amount of skin provided by the skin expansion. The maximum principal stress reached at each expansion is an important data point, since this is associated with the discomfort or pain of the patient during the expansion. The results are presented in the following figures and tables. The boundary was considered simply connected and free to rotate. This boundary condition was chosen after careful observation of the expanded skin to ensure that the skin at the boundary did not exhibit peeling.

Skin is considered homogenous and hyperelastic, though it possesses properties that are much more complex. For example, skin exhibits viscoelastic behavior, which was demonstrated by the decrease in pressure of the expander to zero within one week after the expansion. The contact between the skin and the expander was not considered in the numerical model. This type of material is characterized through the Strain Energy Density, $W$, which is written as a function of the strain invariants $I_{1}, I_{2}$, and $I_{3}$. There 
are several strain energy densities with those characteristics, including Mooney-Rivlin, Neo-Hookean, Ogden, Polynomial, Fung's, Delfino's exponential function, and Ogden [2003]. After assessing these equations as a description of the in vivo results numerically, Delfino's exponential function was chosen, since it provided the best fit for these data. This equation was initially proposed to describe a human artery under several loads and is represented

$$
W=\frac{a}{b}\left\{\exp \left[\frac{b}{2}\left(I_{1}-3\right)\right]-1\right\},
$$

where $a$ and $b$ are parameters of the material and $I_{1}$ is the first strain invariant, defined in terms of the principal stretches $\lambda_{i}$ by

$$
I_{1}=\lambda_{1}^{2}+\lambda_{2}^{2}+\lambda_{3}^{2}
$$

For incompressible materials, such as biological tissues, the third invariant $I_{3}=1$ is used, given that the ratio between the initial thickness, $H$, and final thickness, $t$, of each expansion is equal to the product of the principal stretches:

$$
\lambda_{3}=\frac{t}{H}=\frac{1}{\lambda_{1} \lambda_{2}}
$$

To analyze the numerical results for parameters $a$ and $b$, two variables are used: the ratio $V_{i}^{*}$, and the ratio between the initial thickness, $H$, and final medium thickness, $t$.

\subsection{Numerical results for the scalp.}

Patient 1. A mesh of 126 quadrilateral finite membrane elements, M3D4, was used on the rectangular skin expander of Patient 1, for which the dimensions were $13.6 \mathrm{~cm} \times 5.5 \mathrm{~cm}$ and $V_{\exp }=400 \mathrm{ml}$.

For each expansion, a curve relating the inserted volume and the pressure inside the expander was calculated, which identifies the parameters that best describe the skin at each specific expansion. The Newton Raphson method was used to fit the numerical and in vivo results. Figure 1 shows the numerical and in vivo curves for the sixth expansion of the first patient with the fitting parameters $a=1.79 \mathrm{MPa}$ and $b=120.5$ of Delfino's exponential function. The data indicate that the maximum difference between the

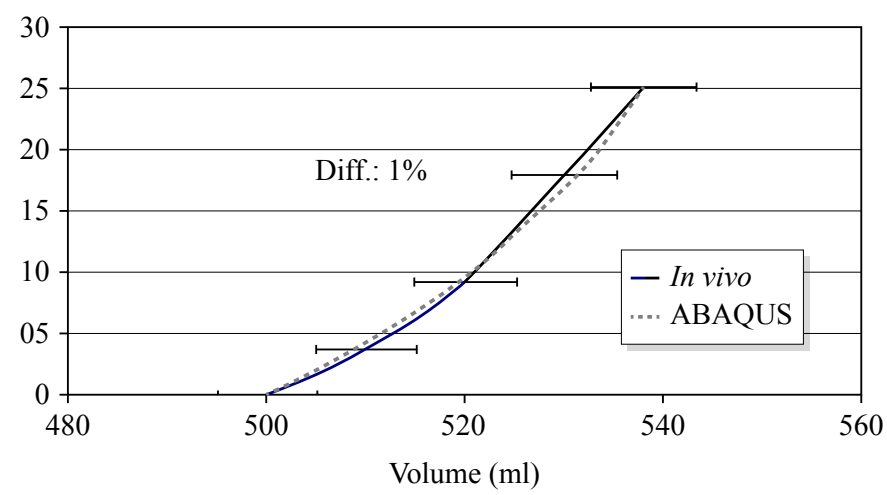

Figure 1. The relationship between the measured pressure $(\mathrm{kPa})$ and inserted volume (ml) for the in vivo and numerical data of the sixth expansion of patient 1 using parameters $a=1.79 \mathrm{MPa}$ and $b=120.5$. 


\begin{tabular}{|cccccccccccc|}
\hline$(i)$ & $V_{i-1} \mathrm{ml}$ & $V_{i} \mathrm{ml}$ & $V_{i}^{*}$ & $P_{i} \mathrm{kPa}$ & $\lambda_{\max }$ added & $\sigma_{\max } \mathrm{kPa}$ & $t \mathrm{~cm}$ & $H / t$ & $a \mathrm{MPa}$ & $b$ \\
$\mathrm{~A}$ & 0 & 80 & $\mathrm{x}$ & $\mathrm{x}$ & $\mathrm{x}$ & $\mathrm{x}$ & $\mathrm{x}$ & & $\mathrm{x}$ & $\mathrm{x}$ \\
1 & 80 & 110 & 0.28 & 29.50 & 1.50 & 241.52 & 0.33 & 1.52 & 0.21 & 31.5 \\
2 & 110 & 140 & 0.35 & 28.20 & 1.66 & 257.93 & 0.29 & 1.72 & 0.22 & 33.4 \\
$\mathrm{~B}$ & 140 & 380 & $\mathrm{x}$ & $\mathrm{x}$ & $\mathrm{x}$ & $\mathrm{x}$ & $\mathrm{x}$ & $\mathrm{x}$ & $\mathrm{x}$ & $\mathrm{x}$ \\
3 & 380 & 425 & 1.06 & 26.10 & 3.15 & 501.88 & 0.16 & 3.16 & 0.99 & 51.3 \\
4 & 425 & 465 & 1.16 & 26.80 & 3.34 & 604.67 & 0.15 & 3.33 & 1.30 & 75.2 \\
5 & 465 & 500 & 1.25 & 26.00 & 3.51 & 642.14 & 0.14 & 3.57 & 1.46 & 134.5 \\
6 & 500 & 538 & 1.35 & 25.20 & 3.65 & 679.23 & 0.13 & 3.85 & 1.79 & 120.5 \\
\hline
\end{tabular}

Table 5. Results of expansion for Patient 1. For expansion $(i): V_{i-1}=$ initial volume; $V_{i}=$ final volume; $V_{i}^{*}=V_{i} / V_{\text {exp }} ; t=$ final thickness.

numerical and in vivo results was $1 \%$. The calculation of a volume versus pressure curve was calculated for each patient at every expansion point, which allows for the characterization of skin at each stage of the stretch.

Using the numerical analysis described, it is possible to fit the results of the numerical and in vivo analysis of the first expansion with the parameters $a=0.21 \mathrm{MPa}$ and $b=31.5$ of Delfino's exponential function. Figure 2 shows the principal stresses that occur during several stages of the expansion. Table 5 shows parameters $a$ and $b$ of Delfinos's function obtained from all the expansions of this patient. Parameter $a$ changed from $0.21 \mathrm{MPa}$ to $1.79 \mathrm{MPa}$ and parameter $b$ changed from 31.5 to 120.5 . In addition, the initial thickness of $0.5 \mathrm{~cm}$ decreased to a final thickness of $0.13 \mathrm{~cm}$, and the final thickness was expected to be smaller than the initial thickness, since the mean value of the thickness was used as the expansions progressed. Moreover, the maximum total stretch, $\lambda_{\max }$, reached 3.65 and the maximum principal stress, $\sigma_{\max }$, reached $679.6 \mathrm{kPa}$ in Patient 1.

Patient 2. A mesh of 161 triangular finite membrane elements, M3D3, was used on the crescent skin expander for Patient 2, with dimensions of $10.1 \mathrm{~cm} \times 5.6 \mathrm{~cm}$ and $V_{\exp }=300 \mathrm{ml}$. Using the numerical analysis described, we fit the results of the numerical and in vivo analysis from expansion one using the parameters $a=0.64 \mathrm{MPa}$ and $b=42.6$ of Delfino's exponential function. We found that parameter $a$ changed from $0.64 \mathrm{MPa}$ to $1.50 \mathrm{MPa}$ and parameter $b$ changed from 42.6 to 65.6 (Table 6). The initial

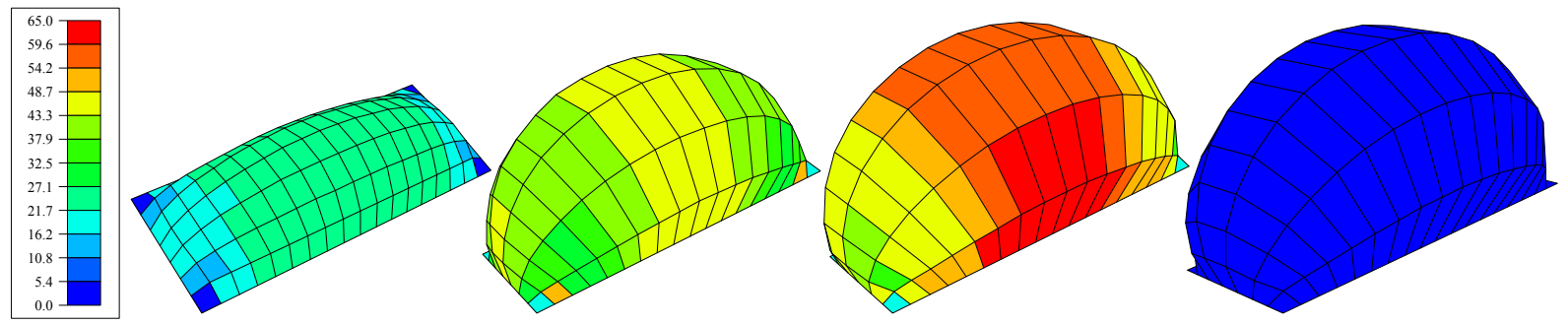

Figure 2. Finite element results for the principal stresses for patient $1\left(V_{\exp }=400 \mathrm{ml}\right)$ with, 126 quadrilateral finite membrane elements, M3D4: (a) 80-110 ml; (b) 380$425 \mathrm{ml}$; (c) $500-538 \mathrm{ml}$; (d) $500-538 \mathrm{ml}$ after 7 days. 


\begin{tabular}{|cccccccccccc|}
\hline$(i)$ & $V_{i-1} \mathrm{ml}$ & $V_{i} \mathrm{ml}$ & $V_{i}^{*}$ & $P_{i} \mathrm{kPa}$ & $\lambda_{\max }$ added & $\sigma_{\max } \mathrm{MPa}$ & $t \mathrm{~cm}$ & $H / t$ & $a \mathrm{MPa}$ & $b$ \\
$\mathrm{~A}$ & 0 & 214 & $\mathrm{x}$ & $\mathrm{x}$ & $\mathrm{x}$ & $\mathrm{x}$ & $\mathrm{x}$ & & $\mathrm{x}$ & $\mathrm{x}$ \\
1 & 214 & 254 & 0.85 & 26.00 & 3.92 & 1,17 & 0.10 & 5.00 & 0.64 & 42.6 \\
2 & 254 & 298 & 0.99 & 25.10 & 4.24 & 1,25 & 0.09 & 5.56 & 0.49 & 48.2 \\
3 & 298 & 338 & 1.13 & 23.90 & 4.49 & 1,08 & 0.08 & 6.25 & 1.01 & 49.8 \\
4 & 338 & 376 & 1.25 & 21.90 & 4.72 & 1,06 & 0.07 & 7.14 & 1.50 & 65.6 \\
\hline
\end{tabular}

Table 6. Results of the parameters assessed for Patient 2.

thickness of $0.5 \mathrm{~cm}$ decreased to a final thickness of $0.07 \mathrm{~cm}$, since the final value should be even smaller because we used the mean value for the thickness as the expansions progressed. The maximum stretch, $\lambda_{\max }$, reached 4.72 and the maximum principal stress, $\sigma_{\max }$, reached $1.06 \mathrm{MPa}$ in Patient 2.

The data indicated that a principal stress, $\sigma_{\max }$, achieved during each skin expansion ranged between 679.6-1,060 kPa. This maximum stress is related with the pressure inside the skin expander, limited by the discomfort or pain felt by the patient.

\subsection{Numerical results for the skin of the lower leg.}

Patient 3. A mesh of 104 quadrilateral finite membrane elements, M3D4, was used on the rectangular skin expander of Patient 3, Figure 3, for which the dimensions were $9.6 \mathrm{~cm} \times 5.9 \mathrm{~cm}$ and $V_{\exp }=250 \mathrm{ml}$. Using the numerical analysis described, we fit the results of the numerical and in vivo analysis and found that parameter $a$ changed from $0.04 \mathrm{MPa}$ to $0.30 \mathrm{MPa}$ and parameter $b$ changed from 22.0 to 45.0 (Table 7). The initial thickness of $0.80 \mathrm{~cm}$ decreased to a final thickness of $0.18 \mathrm{~cm}$ after expansion. The maximum stretch, $\lambda_{\max }$, reached 3.00 and the maximum principal stress, $\sigma_{\max }$, was $131.90 \mathrm{kPa}$ in Patient 3.

Patient 4. A mesh of 126 quadrilateral finite membrane elements (M3D4) was used on the rectangular skin expander of Patient 4 , for which the dimensions were $13.6 \mathrm{~cm} \times 5.5 \mathrm{~cm}$ and $V_{\exp }=400 \mathrm{ml}$. Using the numerical analysis described, it was possible to fit the results of the numerical and in vivo analyses together. Table 8 shows parameters $a$ and $b$ for Delfino's exponential function for this patient. Parameter

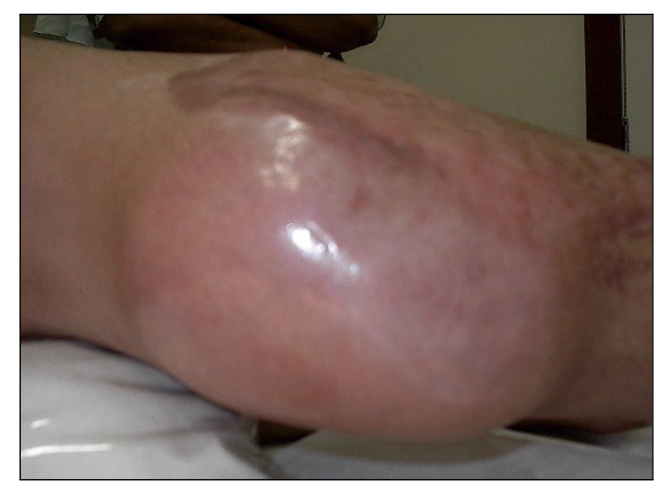

Figure 3. Final expansion of patient 3; as the skin is expanded, the thickness of both dermis and epidermis decreases. 


\begin{tabular}{|ccccccccccc|}
\hline$(i)$ & $V_{i-1} \mathrm{ml}$ & $V_{i} \mathrm{ml}$ & $V_{i}^{*}$ & $P_{i} \mathrm{kPa}$ & $\lambda_{\max }$ added & $\sigma_{\max } \mathrm{kPa}$ & $t \mathrm{~cm}$ & $H / t$ & $a \mathrm{MPa}$ & $b$ \\
$\mathrm{~A}$ & 0 & 157 & $\mathrm{x}$ & \multicolumn{1}{c}{$\mathrm{x}$} & $\mathrm{x}$ & $\mathrm{x}$ & $\mathrm{x}$ & $\mathrm{x}$ & $\mathrm{x}$ & $\mathrm{x}$ \\
1 & 157 & 187 & 0.75 & 2.63 & 2.38 & 27.70 & 0.27 & 2.96 & 0.04 & 22.00 \\
2 & 187 & 232 & 0.93 & 13.00 & 2.64 & 143.80 & 0.23 & 3.48 & 0.08 & 32.80 \\
3 & 232 & 272 & 1.09 & 10.50 & 2.86 & 136.10 & 0.20 & 4.00 & 0.16 & 32.90 \\
4 & 272 & 302 & 1.21 & 9.00 & 3.00 & 131.90 & 0.18 & 4.44 & 0.30 & 45.00 \\
\hline
\end{tabular}

Table 7. Results for parameters assessed in Patient 3.

\begin{tabular}{|ccccccccccc|}
\hline (i) & $V_{i-1} \mathrm{ml}$ & $V_{i} \mathrm{ml}$ & $V_{i}^{*}$ & $P_{i} \mathrm{kPa}$ & $\lambda_{\max }$ added & $\sigma_{\max } \mathrm{kPa}$ & $t \mathrm{~cm}$ & $H / t$ & $a \mathrm{MPa}$ & $b$ \\
$\mathrm{~A}$ & 0 & 120 & $\mathrm{x}$ & $\mathrm{x}$ & $\mathrm{x}$ & $\mathrm{x}$ & $\mathrm{x}$ & $\mathrm{x}$ & $\mathrm{x}$ & $\mathrm{x}$ \\
1 & 120 & 160 & 0.40 & 17.20 & 1.86 & 94.30 & 0.47 & 1.70 & 0.10 & 12.90 \\
2 & 160 & 200 & 0.50 & 18.90 & 2.09 & 128.40 & 0.41 & 1.95 & 0.09 & 27.20 \\
$\mathrm{~B}$ & 200 & 240 & $\mathrm{x}$ & $\mathrm{x}$ & $\mathrm{x}$ & $\mathrm{x}$ & $\mathrm{x}$ & $\mathrm{x}$ & $\mathrm{x}$ & $\mathrm{x}$ \\
3 & 240 & 280 & 0.70 & 12.90 & 2.50 & 114.50 & 0.34 & 2.35 & 0.13 & 36.50 \\
4 & 280 & 320 & 0.80 & 12.60 & 2.70 & 123.70 & 0.31 & 2.58 & 0.17 & 44.20 \\
\hline
\end{tabular}

Table 8. Results for parameters assessed for Patient 4.

$a$ changed from $0.10 \mathrm{MPa}$ to $0.17 \mathrm{MPa}$, and parameter $b$ changes from 12.9 to 44.2 from expansion 1 to 4 , respectively. In addition, the initial thickness of $0.8 \mathrm{~cm}$ decreased to a final thickness of $0.31 \mathrm{~cm}$. The maximum stretch, $\lambda_{\max }$, reached 2.7 and the maximum principal stress, $\sigma_{\max }$, was $123.4 \mathrm{kPa}$.

As seen in Tables 7 and 8, the results showed that a principal stress, $\sigma_{\max }$, achieved during each skin expansion of the lower leg was of the same magnitude and ranged between 144-128 kPa, which was lower than that obtained on the scalp.

4.3. Comparison between the scalp and skin of the lower leg related to $V^{*}$. We next assessed the change in parameters $a$ and $b$ in relation to volume $V^{*}$ as the skin was expanded. To obtain the change in the elastic parameters $a$ and $b$ of Delfino's exponential function for the scalp and the skin of the lower leg, the following procedure was used. Figure 4 shows the relationship between $V_{i}^{*}$ and parameters $a$ and $b$, obtained from Tables 5, 6, 7, and 8 for each skin expansion $i$.

The pressures measured in vivo at the beginning of each expansion were very small, so it was very difficult to obtain accurate measurements. Therefore, the results obtained for the parameters $a$ and $b$ can be considered as an important first step to understanding the behavior of the skin as it is expanded by taking into account differences between the patients in this study in terms of skin site, age, and race. The behavior of the parameters $a$ and $b$ during skin expansion of the scalp as a function of $V^{*}$ is described by

$$
a=0.088+0.006 e^{4.25 V^{*}}, \quad b=27.66+0.06 e^{5.46 V^{*}} .
$$

These equations can predict the changes of the parameters for the skin of the scalp as it is stretched. The parameters for skin stretched for the first time $V^{*}=0$ were $a=0.094 \mathrm{MPa}$ and $b=27.72$, and increased exponentially as the expansion progressed. 

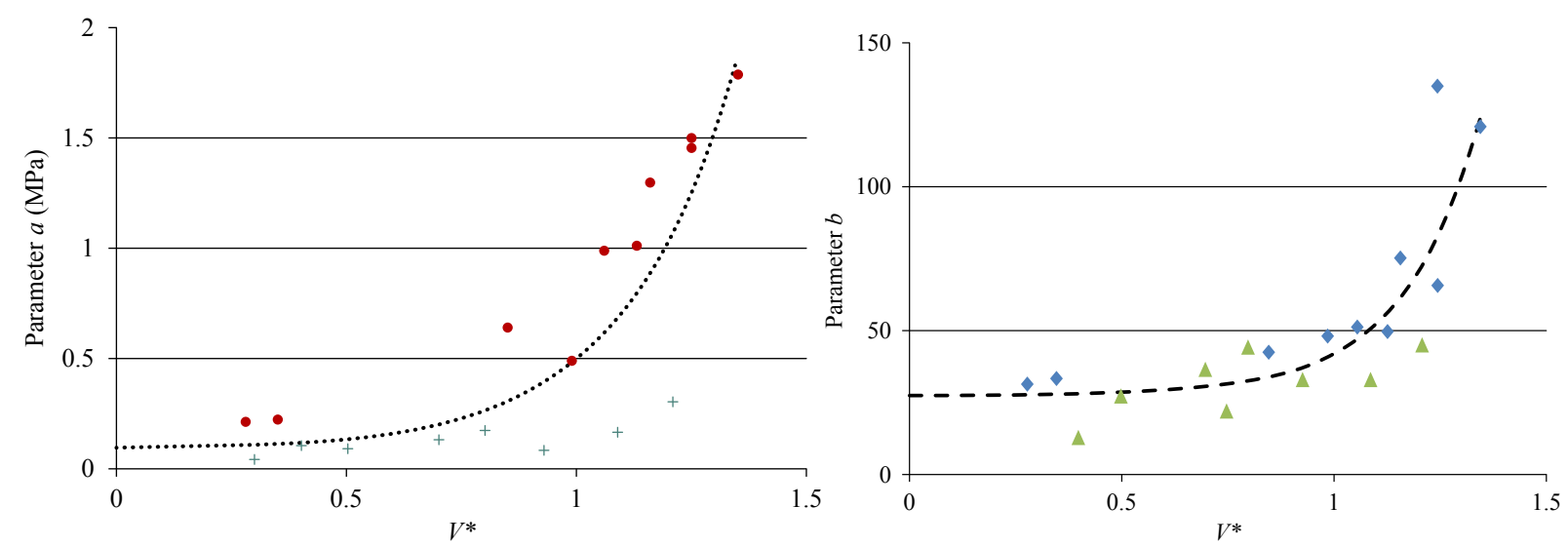

Figure 4. Continuous curves describing the change in parameters $a$ (left) and $b$ (right) with the variable $V^{*}$ for skin expansion of both the scalp and lower leg. For unstretched skin, $a$ was $0.094 \mathrm{MPa}$. For unstretched skin of the lower leg, $b$ was 27.72 .

4.4. Comparison of the thickness ratio between the scalp and skin of the lower leg. The results of this study could potentially be useful for plastic surgeons in various cosmetic procedures if the parameters $a$ and $b$ obtained could be connected to the ratio between the original thickness, $H$, and the thickness after expansion, $t$ [Pitanguy et al. 1998]. When we combined the results in this manner, it became clear that the parameters of the first patient that received skin expansion of the scalp did not fit well with the other three patients, as seen in Figure 5. Specifically, the left half of the figure shows the behavior of $a$ for patient 1 compared to the other 3 patients. When $\lambda_{1} \lambda_{2}=H / t=1$, there was no deformation, and the value of $a$ for the scalp of patient 1 was $0.12 \mathrm{MPa}$, as a particular case of the fit

$$
a=0.045 e^{0.98 \lambda_{1} \lambda_{2}}
$$

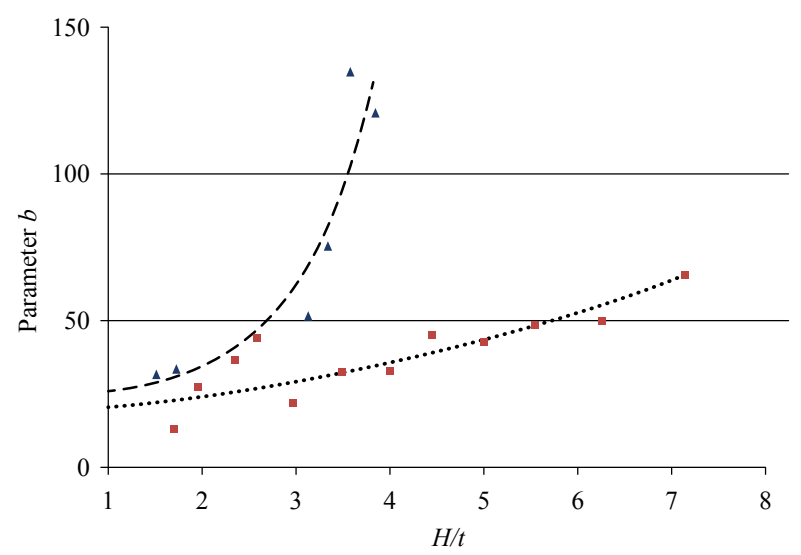

Figure 5. Changes in parameters $a$ (left) and $b$ (right) with the product $\lambda_{1} \lambda_{2}$ for patient 1 (dashed lines) and the other three patients combined (dotted line). 
while for the other three patients combined the value was $0.04 \mathrm{MPa}$ :

$$
a=0.030 e^{0.56 \lambda_{1} \lambda_{2}}-0.01
$$

Figure 5, right, shows the behavior of parameter $b$ for patient 1 compared to the other three patients. When $\lambda_{1} \lambda_{2}=\frac{H}{t}=1$, the value of $b$ for the scalp of patient 1 was 26.50 , as a particular case of the fit

$$
b=22.97+e^{1.26 \lambda_{1} \lambda_{2}}
$$

while for the other three patients combined, it was 20.19:

$$
b=16.71 e^{0.19 \lambda_{1} \lambda_{2}} \text {. }
$$

Although the curves for patient 1 were quite different from the other three patients, the results for parameter $b$ (26.5 and 20.2 for patient 1 and the other 3 patients combined, respectively) were similar when the skin was first stretched (i.e., $\lambda_{1} \lambda_{2}=1$ ). In contrast, parameter $a$ was three times higher for patient 1 when the skin was first stretched than the other three patients combined $(0.12 \mathrm{MPa}$ vs. $0.04 \mathrm{MPa}$, respectively). Patient 1 being older than the others, with consequent skin flaccidity, is a possible explanation for the results observed. On the other hand, skin expanders used on the scalp are inserted under the galea, which is a tight and inelastic tendon. This procedural difference may also explain the difference in the two curves. The fact that patient 1 had darker skin was not considered as a relevant factor.

\section{Conclusions}

The study described here has pioneered the approach for modeling human skin over successive skin expansions. Based on these results, we have obtained different parameters for characterizing skin as the expansions proceed and the skin is stretched. The characterization of skin located in the scalp and lower leg was possible through the association of the numerical and in vivo analyses. The in vivo measurements showed that the skin relaxed after each expansion, since all pressure measurements inside the expanders were reduced to zero within one week after the procedure. Although there are recent proposals supporting that the tensile stress or the control of the expander internal pressure stimulates the cellular growth, in reality the external control parameter is the volume infiltrated. As a result of the viscoelastic property of the skin, the pressure inside the expander, due to relaxation, drops dramatically in the first days and even in the first hours after expansion. This is the principal reason why skin growth was not considered here, relaxation due to viscoelastic properties and not due to structural or molecular changes is used to model the change in the geometry of the skin.

Although the number of patients assessed for each region of the body was low, we measured between four and six expansions for each patient and obtained at least five measurements for each expansion on each patient. Therefore, the total data obtained from this study included more than 100 volume $\times$ pressure in vivo measurements.

The results presented here are considered reliable, and the parameters of Delfino's exponential function for the skin of three of the patients were $a=40.0 \mathrm{kPa}$ and $b=20.2$. It was not possible to compare our results with the work of other studies because the parameters used when characterizing skin are often from the Elasticity Modulus E, Ogden, Fung's, and other constitutive equations. Those equations and 
many others were considered for our study but did not fit our in vivo data, since the rapid stiffening that occurs when large loads are applied is best described by a constitutive model based on an exponential strain energy formulation. One study used Mooney's equation for the characterization of the nonlinear mechanical behavior of human dermis and found $C_{10}$ to be $9.4 \pm 3.6 \mathrm{kPa}$ and $C_{11}$ to be $82 \pm 60 \mathrm{kPa}$ [Hendriks et al. 2003]. In another study, an equivalent equation to Delfino's equation for the artery was used, and the parameters were found to be $a=44.2 \mathrm{kPa}$ and $b=16.7$ [Dorfmann et al. 2010].

The results presented here used two different correlations, and parameters were analyzed in relation to the ratios of $V^{*}$ and $H / t$. The curves obtained for the ratio of $V^{*}$ were well behaved and provided considerable insight of human skin under expansion, which is still an unexplored area of study. However, these data are only useful when analyzing expanded skin. On the other hand, the curves that related parameters $a$ and $b$ with the ratio between the initial and maximum deformed thickness provided important and useful clues of the skin for scientists and surgeons. These analyses allow for the estimation of parameters of skin that is already stretched. After reconstructive surgery, the skin is already stretched, and the results presented here would allow the surgeon to predict the elasticity of the skin after two, three, or even four plastic reconstructive surgeries. Stiffening of the skin after several stretches was seen for both parameters. After the difference in the ratio between thicknesses increased to more than 4-fold, pulling the skin more would not eliminate the wrinkling effect, since it becomes more and more inelastic, which is well documented in the field of cosmetic surgery. As seen empirically, the skin showed an almost rigid body translation, so that pulling the skin in the vicinity of important anatomical structures, such as the eyelids or mouth, may indeed cause deformation of these structures. Importantly, this observation is seen in repeated facial plastic surgeries and serial reconstructive procedures.

It is important to note that as skin is extended, such as with expanders or in other procedures that tighten the skin, the collagen fibers are also extended and cause stiffening in the skin, which results in it being more and more resistant to expansion or further stretching. We observed this phenomenon as an increase in parameters $a$ and $b$ of Delfinos's constitutive equation as subsequent expansions continued.

The results presented in this study are very promising in this field and extend our understanding of the expansion of skin and other biological tissues. Additional research will provide the type, number, and volume of skin expanders, as well as frequency of expansions on several anatomic sites, which are factors necessary to obtain a specified amount of skin for the repair of particular medical problems.

\section{List of symbols}

$\begin{array}{ll}V_{\exp } & \text { nominal volume } \\ V_{i-1} & \text { initial volume } \\ V_{i} & \text { final volume }\end{array}$

$\begin{array}{ll}V^{*} & \text { ratio between } V_{i} \text { and } V_{\exp } \\ V^{* *} & \text { ratio between infiltrated volume and } V_{\exp } \\ H & \text { initial thickness of the skin } \\ t & \text { final skin thickness }\end{array}$

Acknowledgments

We are especially grateful to Professor Ivo Pitanguy, Dr. Henrique N. Radwanski, and his staff, who have supported our projects over the years. Santa Casa da Misericórdia General Hospital of Rio de Janeiro and the Ivo Pitanguy Institute of Plastic Surgery approved this research. Financial support from 
Coordination of Improvement for Higher Level Education (CAPES; grant number E: 0567-2006) and the Brazilian Council for Scientific and Technological Research (CNPq; grant number 301832/2009-9) were essential to making this research possible. We also extend special thanks for the agreement, support, and enthusiasm of the patients without whom this work would not have been possible.

\section{References}

[Alexander 1971] H. Alexander, "Tensile instability of initially spherical balloons”, Int. J. Eng. Sci. 9:1 (1971), 151-160.

[Austad and Rose 1982] E. D. Austad and G. L. Rose, "A self-inflating tissue expander", Plast. Reconst. Surg. 70 (1982), 588-594.

[Beauchene et al. 1989] J. G. Beauchene, M. M. Chambers, A. E. Peterson, and P. G. Scott, "Biochemical, biomechanical, and physical changes in the skin in an experimental animal model of therapeutic tissue expansion", J. Surgical Res. 47:6 (1989), 507-514.

[Buganza Tepole et al. 2011] A. Buganza Tepole, C. J. Ploch, J. Wong, A. K. Gosain, and E. Kuhl, "Growing skin: a computational model for skin expansion in reconstructive surgery", J. Mech. Phys. Solids 59:10 (2011), 2177-2190.

[Delalleau et al. 2006] A. Delalleau, J. Gwendal, J.-M. Lagarde, and J.-M. Berghjou, "Characterization of mechanical properties of skin by inverse analysis combined with the indentation test", J. Biomech. 39:9 (2006), 1603-1610.

[Delalleau et al. 2008] G. Delalleau, J.-M. Josse, H. Lagarde, J.-M. Zahouani, and A. Bergheau, "A nonlinear elastic behavior to identify the mechanical parameters of human skin in vivo", Skin Res. Technol. 14:2 (2008), 152-164.

[Delfino et al. 1997] A. Delfino, N. Stergiopulos, J. E. Moore, and J.-J. Meister, "Residual strain effects on the stress field in a thick wall finite element model of the human carotid bifurcation", J. Biomech. 30:8 (1997), 777-786.

[Diridollou et al. 2000] S. Diridollou, F. Patat, F. Gens, L. Vaillant, D. Black, J. M. Lagarde, Y. Gall, and M. Berson, "In vivo model of the mechanical properties of the human skin under suction", Skin Res. Technol. 6:4 (2000), 214-221.

[Dorfmann et al. 2010] A. Dorfmann, C. Wilson, E. S. Edgar, and R. S. Peattie, "Evaluating patient-specific abdominal aortic aneurysm wall stress based on flow-induced loading", Biomech. Model. Mechanobiol. 9:2 (2010), 127-139.

[Duffy and Shuter 1994] J. S. Duffy and M. Shuter, "Evaluation of soft-tissue properties under controlled expansion for reconstructive surgical use", Medical Eng. Phys. 16:4 (1994), 304-309.

[Geerligs et al. 2011] M. Geerligs, L. C. A. van Breemen, G. W. M. Peters, P. A. J. Ackermans, F. P. T. Baaijens, and C. W. J. Oomens, "Skin in vitro indentation to determine the mechanical properties of epidermis", J. Biomech. 44:6 (2011), 1176-1181.

[Gonçalves et al. 2008] P. B. Gonçalves, D. C. Pamplona, and S. R. X. Lopes, "Finite deformations of an initially stressed cylindrical shell under internal pressure", Int. J. Mech. Sci. 50:1 (2008), 92-103.

[Gonçalves et al. 2009] P. B. Gonçalves, R. M. Soares, and D. C. Pamplona, "Nonlinear vibrations of a radially stretched circular hyperelastic membrane", J. Sound Vib. 327:1-2 (2009), 231-248.

[Green and Adkins 1970] A. E. Green and J. E. Adkins, Large elastic deformations, Clarendon Press, Oxford, 1970.

[Hendriks et al. 2003] F. M. Hendriks, D. Brokken, J. van Eemeren, and C. W. J. Oomens, "A numerical-experimental method to characterize the non-linear mechanical behaviour of human skin", Skin Res. Technol. 9:3 (2003), 274-283.

[Holzapfel et al. 2000] G. A. Holzapfel, T. C. Gasser, and R. W. Ogden, "A new constitutive framework for arterial wall mechanics and a comparative study of material models", J. Elasticity 61:1-3 (2000), 1-48.

[Lim et al. 2008] K. H. Lim, S. Jeyapalina, H. N. Ho, C. M. Chew, P. C. Y. Chen, C. L. Teo, and B. H. Lim, "Non-invasive prediction of skin flap shrinkage: a new concept based on animal experimental evidence", J. Biomech. 41:8 (2008), 1668-1674.

[Ogden 2003] R. W. Ogden, Nonlinear elasticity, anisotropy, material stability and residual stresses in soft tissue biomechanics of soft tissue in cardiovascular systems, pp. 65-108, CISM Courses and Lectures Series 441, Udine - Italy, 2003.

[Pailler-Mattei et al. 2009] C. Pailler-Mattei, S. Nicoli, F. Pirot, R. Vargiolu, and H. Zahouani, "A new approach to describe the skin surface physical properties in vivo", Colloids Surf. B 68:2 (2009), 200-206.

[Pamplona and Bevilacqua 1992] D. C. Pamplona and L. Bevilacqua, "Large deformations under axial force and moment load of initially flat membranes”, Int. J. Non-Linear Mech. 27:4 (1992), 639-650. 
[Pamplona et al. 2001] D. C. Pamplona, P. B. Gonçalves, M. Davidovich, and H. I. Weber, "Finite axisymmetric deformations of an initially stressed fluid-filled cylindrical membrane", Int. J. Solids Struct. 38:10-13 (2001), 2033-2047.

[Pamplona et al. 2006] D. C. Pamplona, P. B. Gonçalves, and S. Lopes, "Finite deformations of cylindrical membrane under internal pressure", Int. J. Eng. Sci. 48:6 (2006), 683-696.

[Pitanguy et al. 1998] I. Pitanguy, D. C. Pamplona, H. I. Weber, F. Leta, F. Salgado, and H. Radwanski, "Numerical modeling of facial aging", Plast. Reconst. Surg. 102:1 (1998), 200-204.

[Schmidt et al. 1991] S. C. Schmidt, S. E. Logan, J. M. Hayden, S. T. Ahn, and T. A. Mustoe, "Continuous versus conventional tissue expansion: experimental verification of a new technique”, Plast. Reconst. Surg. 87:1 (1991), 10-15.

[Silver et al. 2003] F. H. Silver, L. M. Siperko, and G. P. Seehra, "Mechanobiology of force transduction in dermal tissue", Skin Res. Technol. 9:1 (2003), 3-23.

[Socci et al. 2007] L. Socci, G. Pennati, F. Gervaso, and P. Vena, "An axisymmetric computational model of skin expansion and growth", Biomech. Model. Mechanobiol. 6:3 (2007), 177-188.

[Zahouani et al. 2009] H. Zahouani, C. Pailler-Mattei, B. Sohm, R. Vargiolu, V. Cenizo, and R. Debret, "Characterization of the mechanical properties of a dermal equivalent compared with human skin in vivo by indentation and static friction tests", Skin Res. Technol. 15:1 (2009), 68-76.

[Zöllner et al. 2012] A. M. Zöllner, A. Buganza Tepole, and E. Kuhl, "On the biomechanics and mechanobiology of growing skin", J. Theor. Biol. 297 (2012), 166-175.

Received 26 Dec 2011. Revised 28 Jun 2012. Accepted 30 Jun 2012.

DJenane C. Pamplona: djenane@puc-rio.br

Laboratório de Membranas and Biomembranas, Departamento de Engenharia Civil, Pontífica Universidade Católica,

Rua Marquês de São Vicente, 225, Gávea, 22451-900 Rio de Janeiro, RJ, Brazil

Claudio R. Carvalho: claudio.uff.civil@gmail.com

Departamento de Engenharia Civil, Universidade Federal Fluminense (UFF), Rua Passo da Pátria, 156, 24210-240 Niterói, RJ, Brazil 


\title{
JOURNAL OF MECHANICS OF MATERIALS AND STRUCTURES
}

\author{
jomms.net
}

\author{
Founded by Charles R. Steele and Marie-Louise Steele \\ EDITORS \\ Charles R. STEele \\ DAVIDE BIGONI \\ Stanford University, USA \\ University of Trento, Italy \\ IWONA JASIUK University of Illinois at Urbana-Champaign, USA \\ YASUHIDE SHINDO Tohoku University, Japan
}

\section{EDITORIAL BOARD}

H. D. Bui École Polytechnique, France

J. P. CARTER University of Sydney, Australia

R. M. CHRISTENSEN Stanford University, USA

G. M. L. GLadWELL University of Waterloo, Canada

D. H. Hodges Georgia Institute of Technology, USA

J. Hutchinson Harvard University, USA

C. HwU National Cheng Kung University, Taiwan

B. L. KARIHALOO University of Wales, UK

Y. Y. KIM Seoul National University, Republic of Korea

Z. Mroz Academy of Science, Poland

D. PAmplona Universidade Católica do Rio de Janeiro, Brazil

M. B. RUBIN Technion, Haifa, Israel

A. N. SHUPIKOV Ukrainian Academy of Sciences, Ukraine

T. TARNAI University Budapest, Hungary

F. Y. M. WAN University of California, Irvine, USA

P. WRIGGERS Universität Hannover, Germany

W. YANG Tsinghua University, China

F. ZIEGLER Technische Universität Wien, Austria

PRODUCTION production@msp.org

SILVIO LEVY Scientific Editor

See http://jomms.net for submission guidelines.

JoMMS (ISSN 1559-3959) is published in 10 issues a year. The subscription price for 2012 is US $\$ 555 /$ year for the electronic version, and \$735/year (+\$60 shipping outside the US) for print and electronic. Subscriptions, requests for back issues, and changes of address should be sent to Mathematical Sciences Publishers, Department of Mathematics, University of California, Berkeley, CA 94720-3840.

JoMMS peer-review and production is managed by EditFLow ${ }^{\circledR}$ from Mathematical Sciences Publishers.

\author{
mathematical sciences publishers \\ http://msp.org/ \\ A NON-PROFIT CORPORATION \\ Typeset in $\mathrm{IAT}_{\mathrm{E}} \mathrm{X}$ \\ Copyright $@ 2012$ by Mathematical Sciences Publishers
}




\section{Journal of Mechanics of Materials and Structures}

\section{Volume 7, No. $7 \quad$ September 2012}

Molecular dynamics-based continuum models for the linear elasticity of nanofilms and nanowires with anisotropic surface effects

Wonbae Kim, Seung Yun Rhee and Máenghyo Cho

Characterization of human skin through skin expansion

Djenane C. Pamplona and Claudio R. Carvalho 641

In-plane dynamic excitation of AAC masonry walls patched with FRP: dynamic testing and analysis

DVIR ELMALICH and ODED RABINOVITCH

Hygrothermal analysis of exponentially graded rectangular plates

ASHRAF M. ZENKOUR 687

Green's function for symmetric loading of an elastic sphere with application to contact problems ALEXeY S. TITOVICH and ANDREW N. NORRIS 701

Multi-hit armour characterisation of metal-composite bi-layers

K. Karthikeyan, B. P. Russell, V. S. Deshpande and N. A. Fleck 